\title{
Mfn2 inhibits proliferation and cell-cycle in Hela cells via Ras-NF-KB signal pathway
}

Xiaowen $\mathrm{Liu}^{1,2^{*}+}$, Jun Sun ${ }^{3+}$, Ping Yuan ${ }^{3}$, Kangquan Shou ${ }^{2 *}$, Yuanhong Zhou ${ }^{2}$, Wenqi Gao ${ }^{2}$, Jin She ${ }^{2}$, Jun Hu², Jun Yang ${ }^{2}$ and Jian Yang ${ }^{2}$

\begin{abstract}
Background: Mitofusin 2 (Mfn2) is outer membrane protein, as the inhibitor of Ras protein. This study aimed to investigate the effect of Mfn2 on cell proliferation, and cell-cycle in Hela cervical carcinoma cell lines.

Methods: After treated with Adv-mfn2 or Adv-control for $48 \mathrm{~h}$ and $60 \mathrm{~h}$, the RNA and protein of Mfn2 in Hela cells were detected by qRT-PCR and western blot. The immunofluorescence assay was performed to observe the expression and sub-location of Mfn2 in Hela cells. The flow cytometry was performed to detect the cell cycle of Hela cells, while western blots were performed to observe the Ras-NF-KB signal pathway. Then, the xenografted cervix carcinoma mouse model was used to confirm the effect of Mfn2 in Hela cells in vivo and the expression of Ras-NF-kB signaling pathway in vivo.
\end{abstract}

Results: In immunofluorescence detection, Mfn2 was located in cytoplasmic, not in the nucleus. In addition, Mfn2 inhibited cell proliferation of Hela cells through reducing PCNA protein expression. Mfn2 induced arrest in G0/ G1 phase of the cell cycle in Hela cells. Meanwhile, Mfn2 reduced Cyclin D1 protein expression. Moreover, Mfn2 decreased the Ras signal pathway proteins such as Myc, NF-kB p65, STAT3 in a dose-dependent manner. Then, the in vivo experiment also confirmed that Mfn2 could inhibit the tumor growth, and depress the Cyclin D1, Ras, Myc, NF-kB p65, Erk1/2 and mTOR protein expression.

Conclusions: Mfn2 could significantly inhibit cell proliferation in Hela cells. It might be acted as an potential anticancer target through inducing cell cycle arrest in human cervical carcinoma cells.

Keywords: Mfn2, Ras, Cervical carcinoma, Cell proliferation, Cell-cycle

\section{Background}

Cell hyper-proliferation has long been considered as an important etiological factor of many cancers, and selfsufficiency proliferation signals are sustaining, such as Ras signal pathway $[1,2]$. Mitofusin 2 (Mfn2) is involved in regulation of cell survival and has been of interest in cancer field [3-6]. It may be involved in cervical cancer

\footnotetext{
*Correspondence: Ixw@ctgu.edu.cn; kangquan@ctgu.edu.cn

${ }^{\dagger}$ Xiaowen Liu and Jun Sun contributed equally and share first authorship

${ }^{\ddagger}$ Xiaowen Liu and Kangquan Shou contributed equally and share correspondence authorship

${ }^{1}$ Hubei Key Laboratory of Tumor Microenvironment and Immunotherapy, Medical College, China Three Gorges University, Yichang, China

${ }^{2}$ Institute of Cardiovascular Research \& Department of Center Experiment Laboratory, the First College of Clinical Medical Science, China Three Gorges University, Yichang 44300, China

Full list of author information is available at the end of the article
}

pathogenesis and might serve as a biomarker of cervical SCC in the future [7]. In our and others studies, Mfn2 could induce Hela cells into mitochondrial apoptosis [8], pancreatic cancer into cell autophagy [9], and breast cancer into DNA methylation [10], as a tumour suppressor in many cancers. Mfn-2 is a mitochondrial outer-membrane protein with GTPase activity involved in mitochondrial fusion and fission and apoptosis regulation [11-13]. $M f n 2$ gene encodes for a 757-amino-acid protein containing a p21Ras domains near the N-ter [14]. The p21Ras domain makes Mfn2 as an anti-Ras protein in VSMCs, and regulating the VSMCs cell-cycle [15]. In human breast cancer, overexpression of Mfn2 inhibited the Ras-ERK1/2 signaling pathway, but with deletion of the p21Ras motif partially reduced the anti-tumor function of Mfn2 [10]. 
In our early studies, we found that PTD4-apoptin fusion protein could upgrade Mfn2 expression in cervical carcinoma cells [16]. Then, we found that when the expression of Mfn2 increased, the Hela cells were induced into apoptosis via mitochondrial pathway [8]. In this study, we aimed to investigate whether Mfn2 was involved in proliferation in Hela cells, and activated Ras signaling pathways to inhibit Hela cells proliferation. Our findings provide a new target of cervical carcinoma and suggest candidates for potential use in cervical carcinoma therapy in the future.

\section{Materials and methods Antibodies}

Antibodies were as follows: Anti-Mfn2 (D2D10) (Cell Signaling, 9482) directed against Mfn2 protein, Ras (Cell Signaling, 3965), Cyclin D1 (Cell Signaling, 2922), p44/42 MAPK (Erk1/2, Cell Signaling, 9102), PCNA (PC10) (Cell Signaling, 2586), Myc (Cell Signaling, 5605), mTOR (Cell Signaling, 2972), STAT3 (Cell Signaling, 9139) and NF-kB p65 GAPDH (G-9) (Santa Cruz, sc-365062, monoclonal, mouse) and $\beta$-actin (Tianjin Sungene Biotech, China) antibodies were used as the loading controls.

\section{Construction of $\mathrm{mfn} 2$ expression adenoviral vector}

Rno-mfn2 precursor DNA (Homo sapiens (human), Gene ID: 9927) was synthesized by Genechem (Shanghai, China). The adenovirus expressing mfn2 (Adv-mfn2), or control adenovirus expressing control (Adv-control) was generated using the AdMax system (Microbix Biosystems, Canada) according to Wang's in 2018 [8].

\section{Cell culture}

The human cervical carcinoma cell line HeLa was purchased from the Chinese Culture Tissue Collection Center (CCTCC, China). The cells were cultured in DMEM (Hyclone, USA) supplemented with 10\% FBS (fetal bovine serum; Hyclone, USA) at $37^{\circ} \mathrm{C}$ and $5 \% \mathrm{CO}_{2}$.

\section{qRT-PCR analysis}

The total RNA from Hela cells was extracted using TRIzol@ Reagent (Invitrogen, USA). Reverse transcription and qRT-PCR were performed as described previously $[8$, 17, 18]. Amplification and detection of specific products were performed with the ABI stepone plus (PE Applied Biosystems). The mfn2 mRNA expression was measured by RevertAid Reverse Transcriptase (Thermo scientific, EP0442) and qPCR Master Mix (Fermentas,K0221), and the GAPDH was used as an internal control. The 2$\Delta \Delta C$ t method was used to measure the realtime PCR Data. The following sequence-specific primers of Mfn2 were as follow: F: 5'-ATCTGTGCCAGCAAGTTGACA$3^{\prime}$ and R: 5'-AAGTGAATCCAGAGCCTCGAC-3'.

\section{CCK-8 test}

The cells, seeded into the $96-$ well plate at 3000 cells per well, were incubated with 50, 100 and $150 \mathrm{pfu} / \mathrm{cell}$ of Mfn2 or as negative control with PBS for 48 and $60 \mathrm{~h}$ in $5 \% \mathrm{CO}_{2}$ at $37^{\circ} \mathrm{C}$ according to Wang's paper in 2018 [8].

\section{Cell cycle}

The cells, seeded into the 6 -well plate at $1 \times 10^{5}$ cells per well, were incubated with $100 \mathrm{pfu} / \mathrm{cell} \mathrm{Adv}-\mathrm{mfn} 2$ or Adv-control of Adv-mfn2 or Adv-control for $60 \mathrm{~h}$ in 5\% $\mathrm{CO}_{2}$ at $37{ }^{\circ} \mathrm{C}$. Cells were then washed twice in ice-cold PBS, stained with Cell Cycle and Apoptosis Analysis Kit (Beyotime, C1052, China), for $30 \mathrm{~min}$ at room temperature and analyzed with a BD FACSort flow cytometer (BD Biosciences, USA). Cell cycle data was analyzed by ModFit LT 3.2 software (Verity Software House, Topsham, USA).

\section{Immunofluorescence}

HeLa cells were seeded in 6-well plates at a ratio of 10,000 cells per well. After $12 \mathrm{~h}, \mathrm{Adv}-\mathrm{mfn} 2$ or Advcontrol was added into the medium of $50 \mathrm{pfu} /$ cell and incubated at $37^{\circ} \mathrm{C}$. After $60 \mathrm{~h}$, the cells were washed 3 times with PBS and fixed with $4 \%$ paraformaldehyde for $10 \mathrm{~min}$ at room temperature. Subsequently, the cells were washed 3 times with PBS and permeabilized for 5 min with PBS containing $0.2 \%$ Triton X-100. Anti$\mathrm{mfn} 2$ antibodies were used to detect the presence and cellular localization of $\mathrm{mfn} 2$ protein in HeLa cells, as recently reported [16]. The appropriate Rhodamineconjugated goat anti-mouse IgG antibodies (Pierce, 31569) were used as secondary antibodies. The cellular nuclei were stained with 4, 6-diamidino-2-phenylindole (DAPI, $1 \mu \mathrm{g} / \mathrm{L}$ in PBS, Roche, 10236276001). The cells were analyzed by means of confocal fluorescence microscopy Fluoview FV50 (Olympus, Japan).

\section{Western blotting}

The Whole cell proteins and tissue proteins from model mouse were extracted by ice-cold SDS lysis buffer. The BCA protein assay kit (Pierce, 23227) was used to determine the protein concentrations according to the manufacturer's instructions. The proteins were fractionated on $12 \%$ and $15 \%$ SDS-polyacrylamide gel and electroblotted onto Immobilon-P PVDF transfer membranes (Millipore, IPVH08130), as recently reported [8]. The blots were incubated with Mfn-2, anti-Ras, Cyclin D1, ERK1/2, PCNA, c-Myc, mTOR, STAT3 and $\mathrm{NF}-\mathrm{\kappa B}$ p65. GAPDH and $\beta$-actin were used as the loading controls. The positive signals were visualized by 
Odyssey ${ }^{\circledR}$ Two-Color Infrared Imaging System (Li-Cor, USA).

\section{Xenografted cervix carcinoma mouse model}

$\mathrm{BALB} / \mathrm{c}$ nude mice (4-5 weeks old, female) were obtained from the Hubei Provincial Center for Disease Control and Prevention (HBCDC, China). $5 \times 10^{5}$ Human cervix carcinoma HeLa cells were collected and injected subcutaneously under alar of nude mice as recently reported [8]. When the tumors were visible, the mice were divided randomly into 2 groups, consisting of 5 tumor-bearing mice per group for a 2-week-treatment with Adv-mfn2 or Adv-control, respectively. The solution samples were infected into the tumor tissue. Every 3 day $5 \times 10^{8} \mathrm{pfu} /$ $\mathrm{ml} \mathrm{Adv-mfn2} \mathrm{or} \mathrm{Adv-control} \mathrm{were} \mathrm{applied} \mathrm{per} \mathrm{mouse} \mathrm{as}$ our last paper [8]. All animal studies were carried out in accordance with the "Guide for the Care and Use of Laboratory Animals" and approved by the Hubei Provincial Center for Disease Control and Prevention (HBCDC, China).

\section{Detection of xenografted tumor growth}

The tumor volume was measured before and after the 2-week-treatment by the following formula: volume $=0.52 \times$ length $\times$ width $^{2}[8,16]$. The tumor volume difference was calculated by the following formula: difference $=$ after volume - before volume. After 2-week-treatment, the tumor tissues were obtained, and prepared for HE stains.

\section{HE assay}

After a 2-week-treatment, respectively, the mice were killed and the tumors were fixed with $4 \%$ paraformaldehyde, and paraffin sections were prepared for carrying out HE assay, as recently reported [17].

\section{Statistics}

The Student's $t$ test was used to determine the statistical significance of data. A p value of less than $0.05\left(^{*}\right)$ or less than $0.01(* *)$ was considered to be significant. Data presented in the figures represent the mean \pm standard error.

\section{Ethics statement}

Our animal studies were carried out with the Guide for the Care and Use of Laboratory Animals of the People's Republic of China in strict compliance. All efforts were made to minimize suffering and all procedures were performed under ethylether anesthesia. The study protocol was approved by the Committee on the Ethics of Animal Experiments of the Chinese Centre for Diseases Control and Prevention.

\section{Results}

Over-expression of Mfn2 in Hela cells

To detect whether the adenovirus took the $\mathrm{mfn} 2$ gene into the cervix carcinoma cells, we uesd the qRT-PCR to obverse the expression of $\mathrm{mfn} 2$ gene in Hela cells. Advmfn 2 or Adv-control was given into the wells with $1 \times 10^{6}$ Hela cells. After incubated for $60 \mathrm{~h}$, the cells were collected, and lysed in ice-cold TRIzol@ Reagent to obtain the total RNA. Figure 1a shows that the adenovirus take the $\mathrm{mfn} 2$ gene into the cells, and increases the expression of $\mathrm{mfn} 2$ gene. Then, we detected the expression of Mfn2 protein in Hela cells. We dissolved the Hela cells which were incubated with Adv-mfn2 or Adv-control for $60 \mathrm{~h}$, and used the western blot to obverse the expression of Mfn2 proteins. We could see that Mfn2 proteins were over-expression in Adv-mfn2 Hela cells (Fig. 1b, c).

\section{Cellular location of Mfn2 in Hela cells}

Mfn2 is considered as the outer membrane protein of the mitochondria [11], so we analyzed the cellular location of Mfn2 in Hela cells. The Hela cells were incubated with $50 \mathrm{pfu} / \mathrm{cell} \mathrm{Adv}-\mathrm{mfn} 2$ or Adv-control for $60 \mathrm{~h}$ in 6-well plate at $37^{\circ} \mathrm{C}$. Then, the Mfn2 antibody was incubated in the wells, as DAPI for cellular nuclear. Across the fluorescence microscope, we examined the cellular location of Mfn2 in Hela cells (Fig. 1d). The Mfn2 proteins were increased in Mfn2 group, and located in the cytoplasmic, not in the nuclear.

\section{Mfn2 inhibits the growth of cervix carcinoma cells}

The CCK- 8 was performed for detecting the relative inhibition rate of Hela cells. To obverse the inhibition of Mfn2 in Hela cells, we set the CCK- 8 to detect the relative inhibition rate of Hela cells. The cells were seeded into the 96-well plates as 3000 cells per well. After $12 \mathrm{~h}$, the mediums of 50, 100 and $150 \mathrm{pfu} / \mathrm{cell}$ of Adv-mfn2 or Adv-control was incubated into the wells. After 0 , 48 and $60 \mathrm{~h}$, the cells were analyzed by CCK- 8 test. We could found that the relative inhibition of Mfn2 in Hela increased, depend on the time and the dose (Fig. 1e, f). $60 \mathrm{~h}$ incubation group had the highest inhibition, as well as the $150 \mathrm{pfu} / \mathrm{cell} \mathrm{Adv}-\mathrm{mfn} 2$. It was suggested that Mfn2 could inhibit Hela cells growth with time and dose dependence.

Proliferating Cell Nuclear Antigen (PCNA) is the major coordinator of faithful and processive replication and DNA repair at replication forks [19]. It is bound up with the cells proliferation. Here, PCNA protein was examined to proof the inhibition of Mfn2 in Hela cells. The expression of PCNA in Mfn2 groups was decreased obviously in western blot result in Fig. 1g dependence on the dose of Mfn2. 


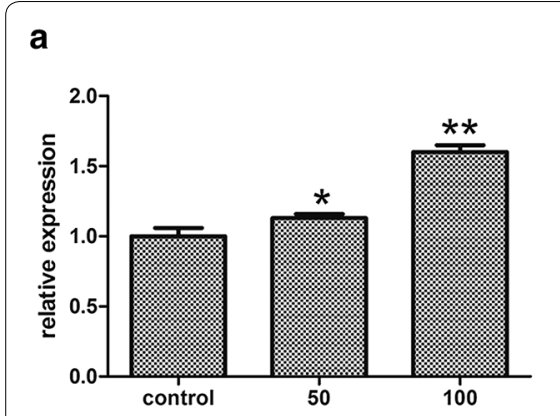

d

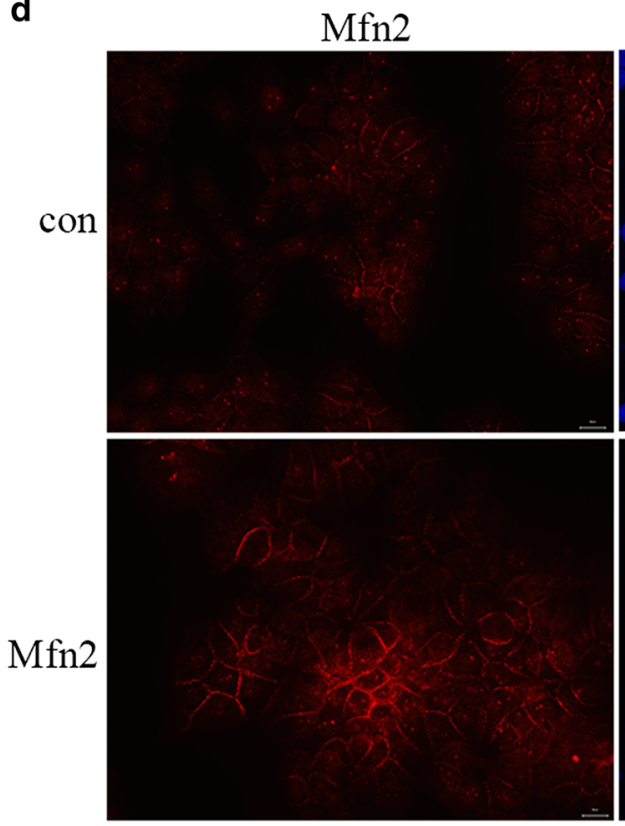

e

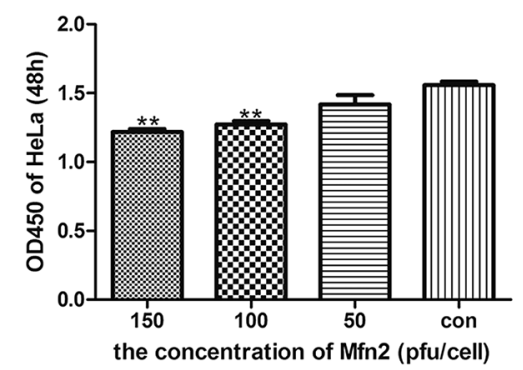

b

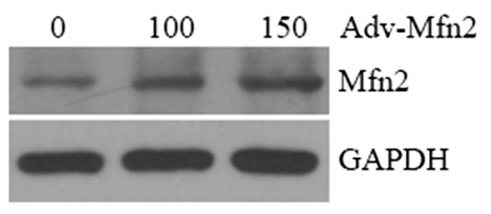

DAPI

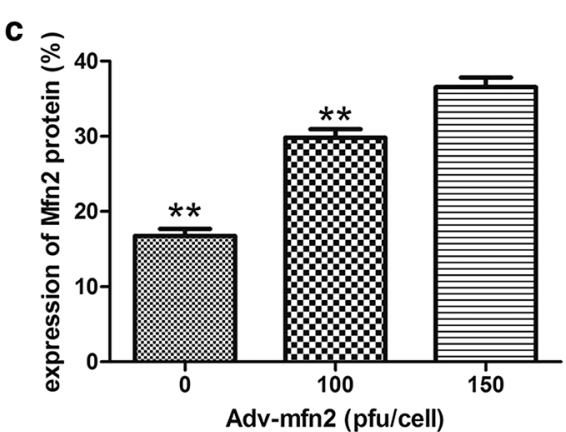

Merge
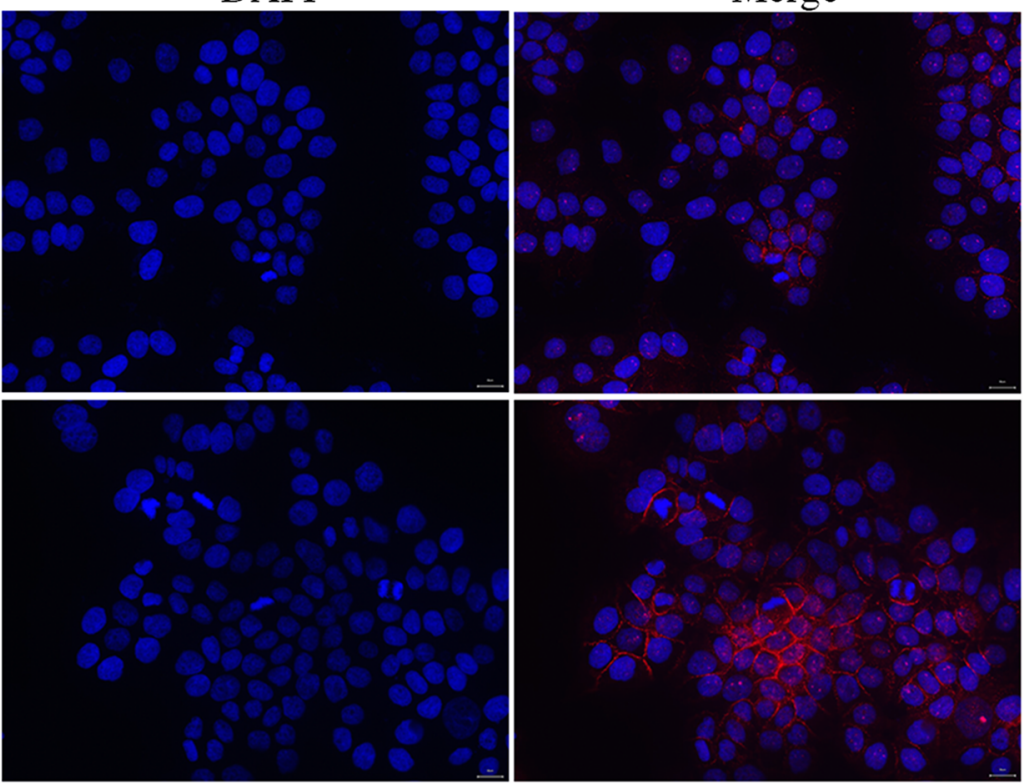

f

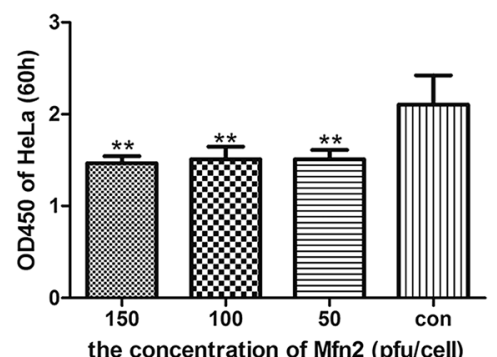

g

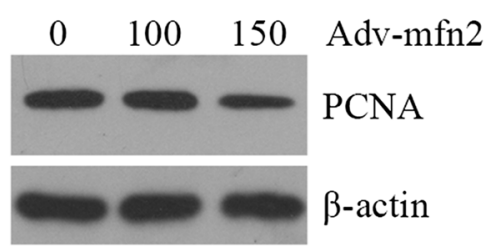

Fig. 1 The overexpression of Mfn2 and its effect on cell proliferation in Hela cells. a HeLa cells were incubated with Ad-Mfn2 (100 or 150pfu/cell) or Adv-control. The expression of Mfn2 mRNA in the Hela cells was detected by qRT-PCR. Data are represented as mean $\pm \operatorname{SD}\left({ }^{*} p<0.05\right.$, $\left.{ }^{* *} p<0.01\right)$. b. c Cell lysate was extracted from HeLa cells treated with Adv-control, Adv-Mfn2 (100 or 150pfu/cell) for Mfn2 expression by western blot analysis (b) and quantitation (c). GAPDH was used as reference. $\mathbf{d}$ Adv-mfn2 were added to the medium of the cells, and incubated for $60 \mathrm{~h}$. Cellular staining of nuclear DNA (DAPI) and localization of Mfn2 protein in HeLa cells. Magnification: 200x. e, $\mathbf{f}$ HeLa cells were exposed to different concentrations of Adv-Mfn2 (0, 50, 100, $150 \mathrm{pfu} / \mathrm{cell})$ for $48 \mathrm{~h}$ and $60 \mathrm{~h}$, and then the cell viability was measured by CCK-8 assay. The Hela cells decreased after being treated with Adv-Mfn2 in a dose- and time-dependent manner. (mean \pm SD from three independent experiments, ${ }^{* *} p<0.01$ ). $g$ After incubated with Adv-mfn2 (0, 100 and 150 pfu/cell), the expression of PCNA protein in HeLa cells was detected by western blot. $\beta$-actin was used as reference

\section{Over-expression of Mfn2 altered cell-cycle in Hela cells} To confirm the possible mechanism of inhibition of Mfn2 in Hela cells, the cell-cycle test was performed in Hela cells incubated with Adv-mfn2. The 100 pfu/cell of Advmfn2 or Adv-control was added into Hela cells in 6-well plate for $60 \mathrm{~h}$. Fluorescence activated cell sorting (FACS) 
analysis was used to examine cell-cycle distribution. Adv-control added into well of Hela cells, approximately 35.53\% of Hela cells infected by Adv-control progressed into $S$ phase. On the contrary, Hela cells infected with Adv-mfn2 remained mostly in the G0/G1 phases with only $26.58 \%$ of cells entering $S$ phase. We speculated that
Mfn2 could further block the Hela cells in G0/G1 phase due to the effects of Adv-mfn2 on Hela cells proliferation as shown in Fig. 2a, b.

Cyclin D1 is an oncogene frequently overexpressed in human cancers that has a dual function as cell cycle and transcriptional regulator, although the latter is widely

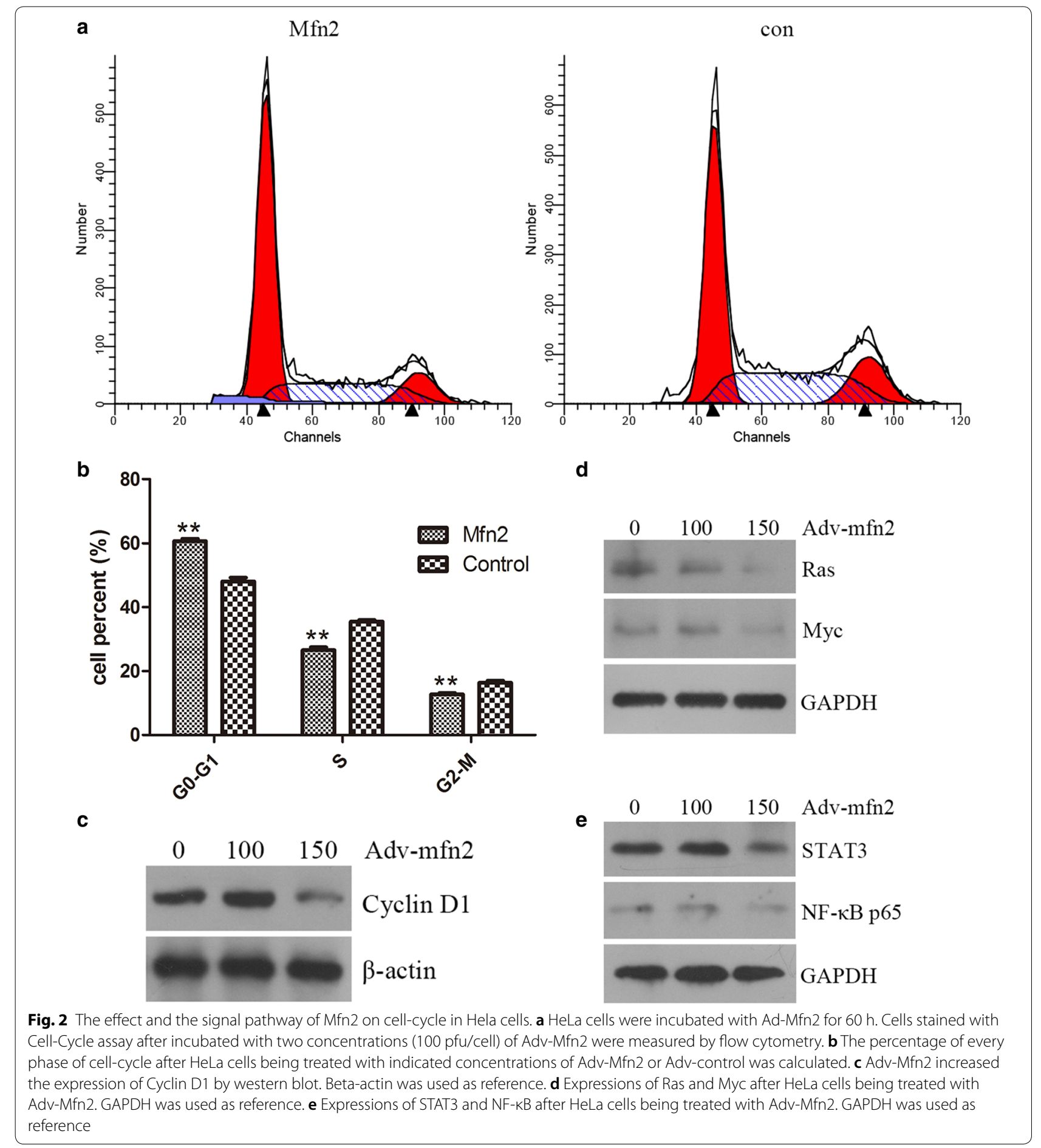


unexplored [18]. Western Blot of Cyclin D1 was used to further confirm that the cell-cycle of Hela cells was inhibited by Cyclin D1 down-expression in Hela cells, depend on the dose of Mfn2 in Fig. 2c.

\section{Mfn2 activity is affected via Ras signal pathway}

The function of the RAS signaling pathway is to integrate extracellular signals and coordinate a suitable response by a subsequent control of cellular growth, survival, and differentiation [20, 21]. Mfn-2 is known to block cell proliferation via inhibition of the Ras pathway in VSMCs $[15,22]$. We examined whether Mfn2-mediated inhibition of cell proliferation and cell cycle in Hela cells was affected by the cellular level of Ras. HeLa cells were incubated with Adv-mfn2 or Adv-control. The results represented in Fig. 2d demonstrate that the expression of Ras are affected by Mfn2. Over-expression of Mfn2 could increase the Ras and Myc protein expression in HeLa cells. Meanwhile, the expressions of STAT3 and NF- $\mathrm{kB}$ p65 protein were also decreased by incubation with AdvMfn2 (Fig. 2d, e).

Therefore, we conclude that Mfn2 inhibits Hele cells proliferation and cell-cycle by activating Ras protein expression. Furthermore, our finding that Myc, STAT3 and NF- $\mathrm{kB}$ p 65 protein are decreased by incubated with Adv-mfn2 in HeLa cells. The present research showed that Ras was sensitive in Mfn2-induced proliferation depressing in HeLa cells, and inactivated its downstream signal pathway as Myc, STAT3 and NF-kB p65 protein expression.

\section{In-vivo, Mfn2 inhibits cervix carcinoma growth}

In order to verify the effect of Mfn2 protein against the cervix carcinoma in vivo, we examined the therapeutic effect of Adv-mfn2 on xenografted cervix carcinoma in a mouse tumor model for a 2-week treatment. HeLa cells were injected subcutaneously and the animals were randomly divided into two groups of each 5 tumor-bearing mice. These tumor-bearing mice were treated with Adv-mfn2 or Adv-control as described in the Materials and Methods section. Before and after the treatment, the volume of the tumors was determined. During the treatment, the volume of xenografted cervix carcinomas treated with Adv-mfn2 increased slowly, whereas the volume of the tumors treated with Adv-control increased faster than the Adv-mfn2 group (Fig. 3a, b). At day 14, the mice were sacrificed and the tumors were macroscopically or histologically analyzed. The tumors treated with Adv-mfn2 were smaller than the ones treated with Adv-control (Fig. 3b). After the 2-week treatment all mice were checked, and there were not any metastases in the mice of two groups.
To detect the underlying mechanism of the Mfn2-triggered tumor reduction, tumor sections of both Adv-mfn2 and Adv-control treated tumors were analyzed by means of a HE assay and Western blot. The nucleus of cervix carcinoma cells treated with Adv-mfn2 was pycnosis and fragmented into several parts in the analyzed histological section. In contrast, the tumors treated with Advcontrol had no signs of nucleus pycnosis (Fig. 3c). Then, Western blot was performed to confirm that Mfn2 was over expression in the tumor tissue of Adv-mfn2 group, and the expression of Cyclin D1 was decreased in Advcontrol group (Fig. 3d-f). The expressions of Ras, Erk1/2, Myc, mTOR and NF- $\mathrm{kB}$ p65 protein in tumor tissues of 2 groups were detected by Western blot, and these were decreased by treated with Adv-mfn2 comparing with the Adv-control group (Fig. 3g, h). We found that Mfn2 could decrease the proteins of Ras-NF-kB signaling pathway.

\section{Discussion}

In the current study, we confirmed that Mfn2 could inhibit Hela cells proliferation in vitro and in vivo, and arrest Hela cells cell-cycle. The cellular location of Mfn2 in Hela cells was performed by immunofluorescence. The results of CCK8 and the western blot of PCNA showed that Mfn2 inhibited the Hela cells proliferation in the dose- and time-dependence. The flow cytometry and the western blot of Cyclin D1 meant that the cell-cycle of Hela cells were arrested by Mfn2 in G0/G1 phase. It was been confirmed that Mfn2 was the inhibitor of Ras in VSMCs [15]. To find the signal pathway of cell-proliferation inhibition and cell-cycle arrest in Mfn2 manner in Hela cells, we detected the Ras, Myc, NF-kB p65, STAT3 proteins by western blot. The results of these western blots suggested that Mfn2 could inhibit the Hela cells by decreasing the expression of Ras and relative proteins in Ras-NF- $\mathrm{kB}$ signal pathway. In xenografted mouse model, we measured the tumor size before and after the Adv$\mathrm{mfn} 2$ or Adv-control treatment, and analyzed the expression of Cyclin D1, Ras, Myc, ERK1/2, NF-kB p65, mTOR proteins by western blot. These results implicated that Mfn2 could inhibit the Hela cells growth by declining the expression of Ras protein and arresting the Ras-NF- $\mathrm{KB}$ signal pathway.

The proliferation of cells was remarkably inhibited, thus inducing the cells into apoptosis or senescence [23, 24]. In our previous study, the inhibition of Mfn2 in Hela cells has been proved to be achieved through the apoptosis induction [8] and cell cycle arrest. Mfn2 could inhibit Ras-Erk1/2 and PI3 k-Akt signal pathway in VSMCs [12, 15]. Ras-Erk1/2 and PI3k-Akt-mTOR are the classic signal pathways of cell proliferation $[25,26]$. NF- $\mathrm{kB}$ has recently generated considerable interest as it has been implicated in human cancer initiation, progression 

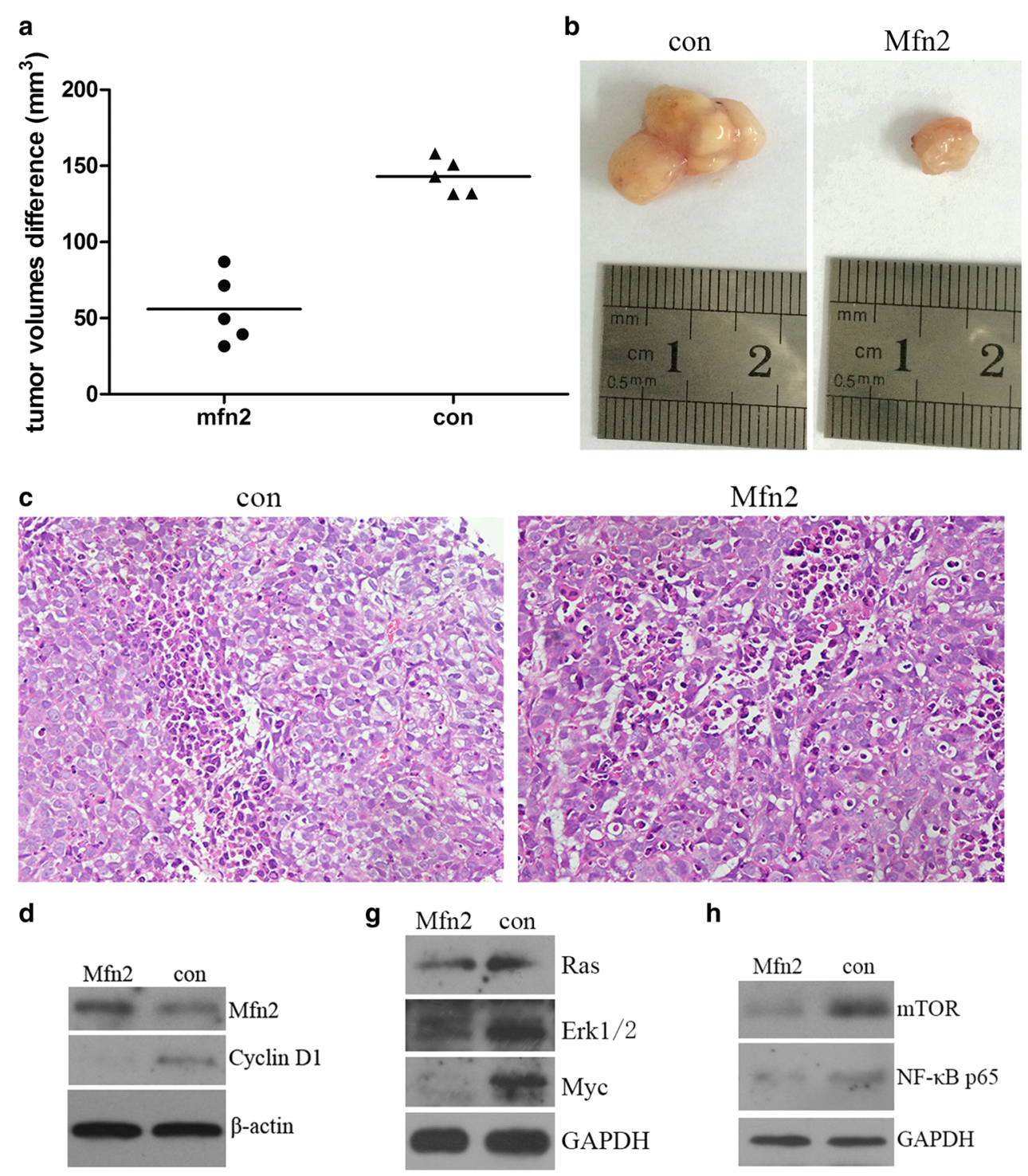

e

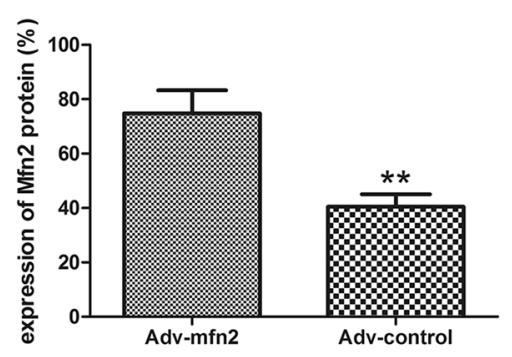

h
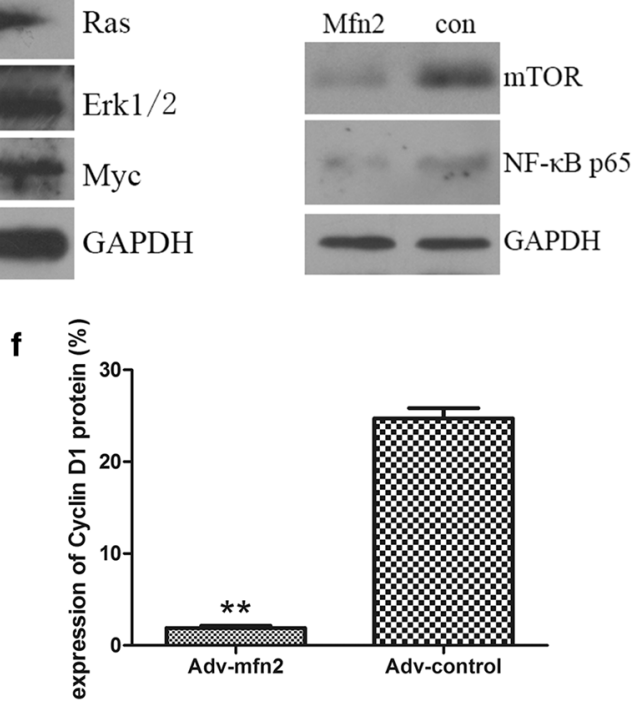

Fig. 3 Mfn2 inhibited the tumor growth in vivo of cervical carcinoma mouse model and its signal pathway. a Determination of the tumor volume in mice treated with Adv-mfn2 or Adv-control for the 0, 3, 6 and 9 days. Data are represented as mean \pm SD (** $p<0.01)$. b The sizes of xenografted cervix carcinoma after 14-days treatment in the Mfn2 group and the con group. $\mathbf{c}$ HE assay of tumor sections from animals treated with either Adv-control or Adv-Mfn2. Magnification: 200x. d-f The expression of Mfn2 and Cyclin D1 proteins in the tumors of mouse model was detected by western blot $(\mathbf{a}, \mathbf{b})$ and quantitation $(\mathbf{c})$. Beta-actin was used as reference. Data are represented as mean $\pm S D\left({ }^{* *} p<0.01\right)$. $\mathbf{g}$ Western blot showed Ras, Erk1/2 and Myc proteins expressions in cervix tumors treated with Adv-mfn2 after 2-weeks treatment. Total tumor tissue lysates were prepared and analyzed by Western blot for these proteins. GAPDH was used as reference. $\mathbf{h}$ Western blot showed mTOR and NF-KB proteins expressions in cervix tumors treated with Adv-mfn2 after 2-weeks treatment. Total tumor tissue lysates were prepared and analyzed by Western blot for these proteins. GAPDH was used as reference 


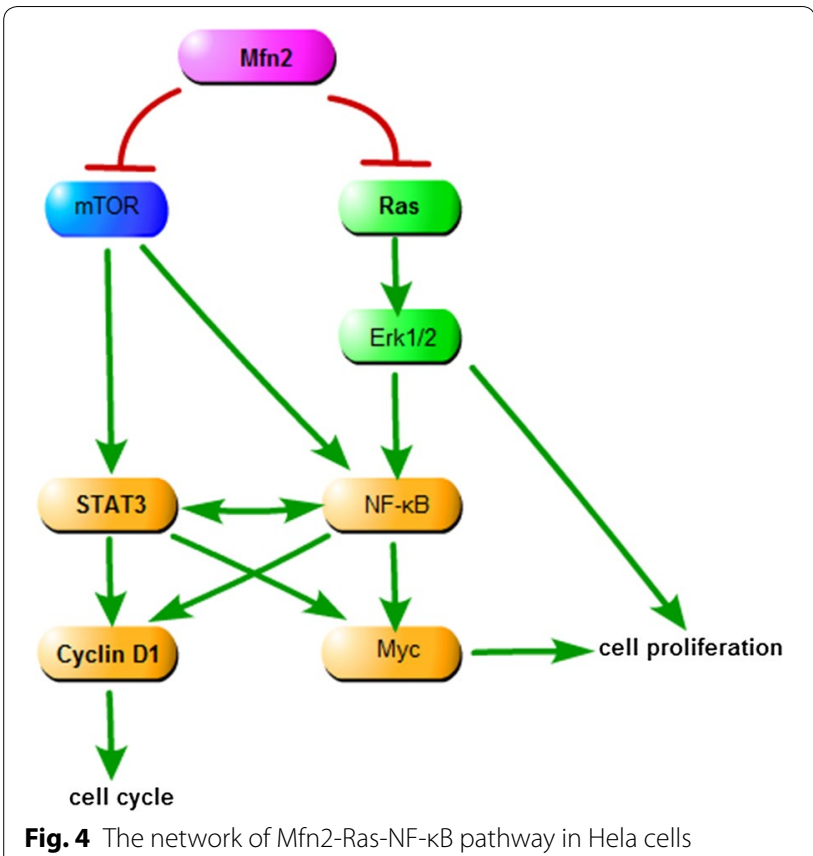

and resistance to treatment [27]. Mutations of upstream signaling molecules, such as Ras, often lead to constitutive activation of NF- $\mathrm{B}$ in solid malignancies $[27,28]$. NF $-\kappa B$ could stimulate the transcription of proliferation regulating genes like Cyclin D1 and Myc [27, 29-31]. NF-kB does not function alone but is part of a network, which determines the pattern of its effects on the expression of STAT3 [32]. Meanwhile, the activation of STAT3 is responsible for genes that promote cell proliferation such as Cylinc D1, Myc and so on [33]. The activation of STAT3 could cause a positive feedback mechanism to NF-kB [34]. STAT3 and NF-kB work together in a network for a result [27]. Therefore, Mfn2 inhibits RasNF- $\mathrm{kB}$ signal pathway to arrest the cell proliferation and cell-cycle in Hela cells (Fig. 4). Based on our research, we propose that Mfn2 may be a novel target to the therapy of cervical carcinoma in the future.

\section{Conclusions}

Mfn2 could inhibit the proliferation and cell cycle in Hela cells. The Ras-NF- $\mathrm{kB}$ signaling pathway was inactive by the expression of Mfn2 increasing. The xenografted cervical carcinoma mouse model was examined to confirm the effect of Mfn2 in Hela cells in vivo.

\section{Abbreviations}

Mfn2: mitofusin 2; FBS: fetal bovine serum; CCK-8: Cell Counting Kit-8.

\section{Acknowledgements}

We thank Jing Zhang, Jiawang Ding, Hui Wu, Chao He, Chun Liu and Chao Luo for helpful discussion.

\section{Authors' contributions}

Conceived and designed the experiments: XWL, JSun, PY. Performed the experiments: XWL, PY, YHZ, WQG, JShe. Analyzed the data: XWL, PY, JY, JY. Contributed reagents/materials/analysis tools: JShe, JH. Contributed to the writing of the manuscript: XWL, PY, JSun, KQS. Advice of the experiments: $J Y, J Y$. Revised the manuscript: KQS. All authors read and approved the final manuscript.

\section{Funding}

National Natural Science Foundation of China for Youth to X. W. Liu. Grant number: 81402568; National Natural Science Foundation of China to J. Sun. Grant number: 81472833; Open fund of Key Laboratory of ischemic cardiovascular and cerebrovascular disease translational medicine (Three Gorges University) to J. She. Grant number: 2017KXN04.

\section{Availability of data and materials}

Not applicable.

\section{Ethics approval and consent to participate}

All applicable international, national, and/or institutional guidelines for the care and use of animals were followed.

\section{Consent for publication}

Not applicable.

\section{Competing interests}

The authors declare that they have no competing interests.

\section{Author details}

${ }^{1}$ Hubei Key Laboratory of Tumor Microenvironment and Immunotherapy, Medical College, China Three Gorges University, Yichang, China. ${ }^{2}$ Institute of Cardiovascular Research \& Department of Center Experiment Laboratory, the First College of Clinical Medical Science, China Three Gorges University, Yichang 44300, China. ${ }^{3}$ Department of Biochemistry and Molecular Biology, Basic Medical College, Tongji Medical College, Huazhong University of Science and Technology, Wuhan, China.

Received: 7 February 2019 Accepted: 19 July 2019

Published online: 29 July 2019

\section{References}

1. Hanahan D, Weinberg RA. Hallmarks of cancer: the next generation. Cell. 2011;144:646-74.

2. Hanahan D, Weinberg RA. The hallmarks of cancer. Cell. 2000;100:57-70.

3. Rocha AG, Franco A, Krezel AM, Rumsey JM, Alberti JM, Knight WC, et al. MFN2 agonists reverse mitochondrial defects in preclinical models of Charcot-Marie-Tooth disease type 2A. Science. 2018;360:336-41.

4. Wang W, Sun Q, Wu Z, Zhou D, Wei J, Xie H, et al. Mitochondrial dysfunction-related genes in hepatocellular carcinoma. Front Biosci (Landmark Ed). 2013;18:1141-9.

5. Sanchis-Gomar F, Derbre F. Mitochondrial fission and fusion in human diseases. N Engl J Med. 2014;370:1073-4.

6. Serasinghe MN, Chipuk JE. Mitochondrial fission in human diseases. Handb Exp Pharmacol. 2017;240:159-88.

7. Ahn SY, Li C, Zhang X, Hyun YM. Mitofusin-2 expression is implicated in cervical cancer pathogenesis. Anticancer Res. 2018;38:3419-26.

8. Wang W, Liu X, Guo X, Quan H. Mitofusin-2 triggers cervical carcinoma cell hela apoptosis via mitochondrial pathway in mouse model. Cell Physiol Biochem. 2018;46:69-81.

9. Xue R, Meng Q, Lu D, Liu X, Wang Y, Hao J. Mitofusin2 induces cell autophagy of pancreatic cancer through inhibiting the PI3K/Akt/mTOR signaling pathway. Oxid Med Cell Longev. 2018;2018:2798070.

10. Li Y, Dong W, Shan X, Hong H, Liu Y, Liu Y, et al. The anti-tumor effects of Mfn2 in breast cancer are dependent on promoter DNA methylation, the P21(Ras) motif and PKA phosphorylation site. Oncol Lett. 2018;15(5):8011-8.

11. Santel A. Get the balance right: mitofusins roles in health and disease. Biochim Biophys Acta. 2006;1763:490-9. 
12. Guo X, Chen KH, Guo Y, Liao H, Tang J, Xiao RP. Mitofusin 2 triggers vascular smooth muscle cell apoptosis via mitochondrial death pathway. Circ Res. 2007:101:1113-22.

13. Schrepfer E, Scorrano L. Mitofusins, from mitochondria to metabolism. Mol Cell. 2016;61:683-94.

14. Chandhok G, Lazarou M, Neumann B. Structure, function, and regulation of mitofusin-2 in health and disease. Biol Rev. 2018;93:933-49.

15. Chen KH, Guo X, Ma D, Guo Y, Li Q, Yang D, et al. Dysregulation of HSG triggers vascular proliferative disorders. Nat Cell Biol. 2004;6:872-83.

16. Liu XW, Yuan P, Tian J, Li LJ, Wang Y, Huang SC, et al. PTD4-apoptin induces $\mathrm{BCl}$-2-insensitive apoptosis in human cervical carcinoma in vitro and in vivo. Anticancer Drugs. 2016;27(10):979-87.

17. Sun J, Yan Y, Wang XT, Liu XW, Peng DJ, Wang M, et al. PTD4apoptin protein therapy inhibits tumor growth in vivo. Int J Cancer. 2009;124:2973-81.

18. Albero R, Enjuanes A, Demajo S, Castellano G, Pinyol M, Garcia N, et al. Cyclin D1 overexpression induces global transcriptional downregulation in lymphoid neoplasms. J Clin Invest. 2018;128(9):4132-47.

19. Slade D. Maneuvers on PCNA rings during DNA replication and repair. Genes (Basel). 2018;9:416

20. Masliah-Planchon J, Garinet S, Pasmant E. RAS-MAPK pathway epigenetic activation in cancer: miRNAs in action. Oncotarget. 2016;7(25):38892-907.

21. Eleveld TF, Schild L, Koster J, Zwijnenburg DA, Alles LK, Ebus ME, et al. RAS-MAPK pathway-driven tumor progression is associated with loss of CIC and other genomic aberrations in neuroblastoma. Cancer Res. 2018;78:6297-307.

22. Chen KH, Dasgupta A, Ding J, Indig FE, Ghosh P, Longo DL. Role of mitofusin 2 (Mfn2) in controlling cellular proliferation. FASEB J. 2014;28(1):382-94.

23. Zanichelli F, Capasso S, Cipollaro M, Pagnotta E, Cartenì M, Casale F, et al. Dose-dependent effects of R-sulforaphane isothiocyanate on the biology of human mesenchymal stem cells, at dietary amounts, it promotes cell proliferation and reduces senescence and apoptosis, while at anti-cancer drug doses, it has a cytotoxic effect. Age. 2012;34:281-93.

24. Nicola Alessio TSSÖ, Venditti MMGP. Stress and stem cells: adult Muse cells tolerate extensive genotoxic stimuli better than mesenchymal stromal cells. Oncotarget. 2018;27:19328-41.
25. Rodenak-Kladniew B, Castro A, Starkel P, De Saeger C, Garcia DBM, Crespo R. Linalool induces cell cycle arrest and apoptosis in HepG2 cells through oxidative stress generation and modulation of Ras/MAPK and Akt/mTOR pathways. Life Sci. 2018;199:48-59.

26. Rosenberg L, Yoon CH, Sharma G, Bertagnolli MM, Cho NL. Sorafenib inhibits proliferation and invasion in desmoid-derived cells by targeting Ras/MEK/ERK and PI3 K/Akt/mTOR pathways. Carcinogenesis. 2018;39:681-8

27. Tilborghs S, Corthouts J, Verhoeven Y, Arias D, Rolfo C, Trinh XB, et al. The role of nuclear factor-kappa B signaling in human cervical cancer. Crit Rev Oncol Hematol. 2017;120:141-50.

28. Chaturvedi MM, Sung B, Yadav VR, Kannappan R, Aggarwal BB. NF-kappaB addiction and its role in cancer: 'one size does not fit all'. Oncogene. 2011:30:1615-30.

29. Perkins ND. Achieving transcriptional specificity with NF-kappa B. Int J Biochem Cell Biol. 1997:29:1433-48.

30. La Rosa FA, Pierce JW, Sonenshein GE. Differential regulation of the c-myc oncogene promoter by the NF-kappa B rel family of transcription factors. Mol Cell Biol. 1994;14:1039-44.

31. Li WM, Han CL, Liu C, Xing HY, Ding DC. ANGPTL2 deletion inhibits osteoclast generation by modulating NF-kappaB/MAPKs/Cyclin pathways. Biochem Biophys Res Commun. 2018;503:1471-7.

32. Hoesel B, Schmid JA. The complexity of NF-kappaB signaling in inflammation and cancer. Mol Cancer. 2013;12:86.

33. Aggarwal BB, Kunnumakkara AB, Harikumar KB, Gupta SR, Tharakan ST, Koca $C$, et al. Signal transducer and activator of transcription-3, inflammation, and cancer: how intimate is the relationship? Ann N Y Acad Sci. 2009;1171:59-76

34. Grivennikov SI, Karin M. Dangerous liaisons: STAT3 and NF-kappaB collaboration and crosstalk in cancer. Cytokine Growth Factor Rev. 2010;21:11-9.

\section{Publisher's Note}

Springer Nature remains neutral with regard to jurisdictional claims in published maps and institutional affiliations.
Ready to submit your research? Choose BMC and benefit from

- fast, convenient online submission

- thorough peer review by experienced researchers in your field

- rapid publication on acceptance

- support for research data, including large and complex data types

- gold Open Access which fosters wider collaboration and increased citations

- maximum visibility for your research: over 100M website views per year

At BMC, research is always in progress.

Learn more biomedcentral.com/submissions 